\title{
Transatlantica
}

Revue d'études américaines. American Studies Journal

\section{0 ans de photographie au New York Times Magazine}

Rencontres d'Arles, été 2011

\section{Anne Lesme}

\section{(2) OpenEdition}

Journals

Édition électronique

URL : http://journals.openedition.org/transatlantica/5367

DOI : $10.4000 /$ transatlantica.5367

ISSN : $1765-2766$

Éditeur

AFEA

Référence électronique

Anne Lesme, « 30 ans de photographie au New York Times Magazine », Transatlantica [En ligne], 1 ।

2011, mis en ligne le 07 janvier 2012, consulté le 29 avril 2021. URL : http://journals.openedition.org/ transatlantica/5367 ; DOI : https://doi.org/10.4000/transatlantica.5367

Ce document a été généré automatiquement le 29 avril 2021

\section{c) (i) $\Theta($}

Transatlantica - Revue d'études américaines est mis à disposition selon les termes de la licence Creative Commons Attribution - Pas d'Utilisation Commerciale - Pas de Modification 4.0 International. 


\section{0 ans de photographie au New York Times Magazine}

Rencontres d'Arles, été 2011

Anne Lesme

NOTE DE L'AUTEUR

Les Photographies du New York Times Magazine, Rencontres d'Arles, du 4 juillet au 4 septembre 2011, chapelle Sainte-Anne, place de la République. 


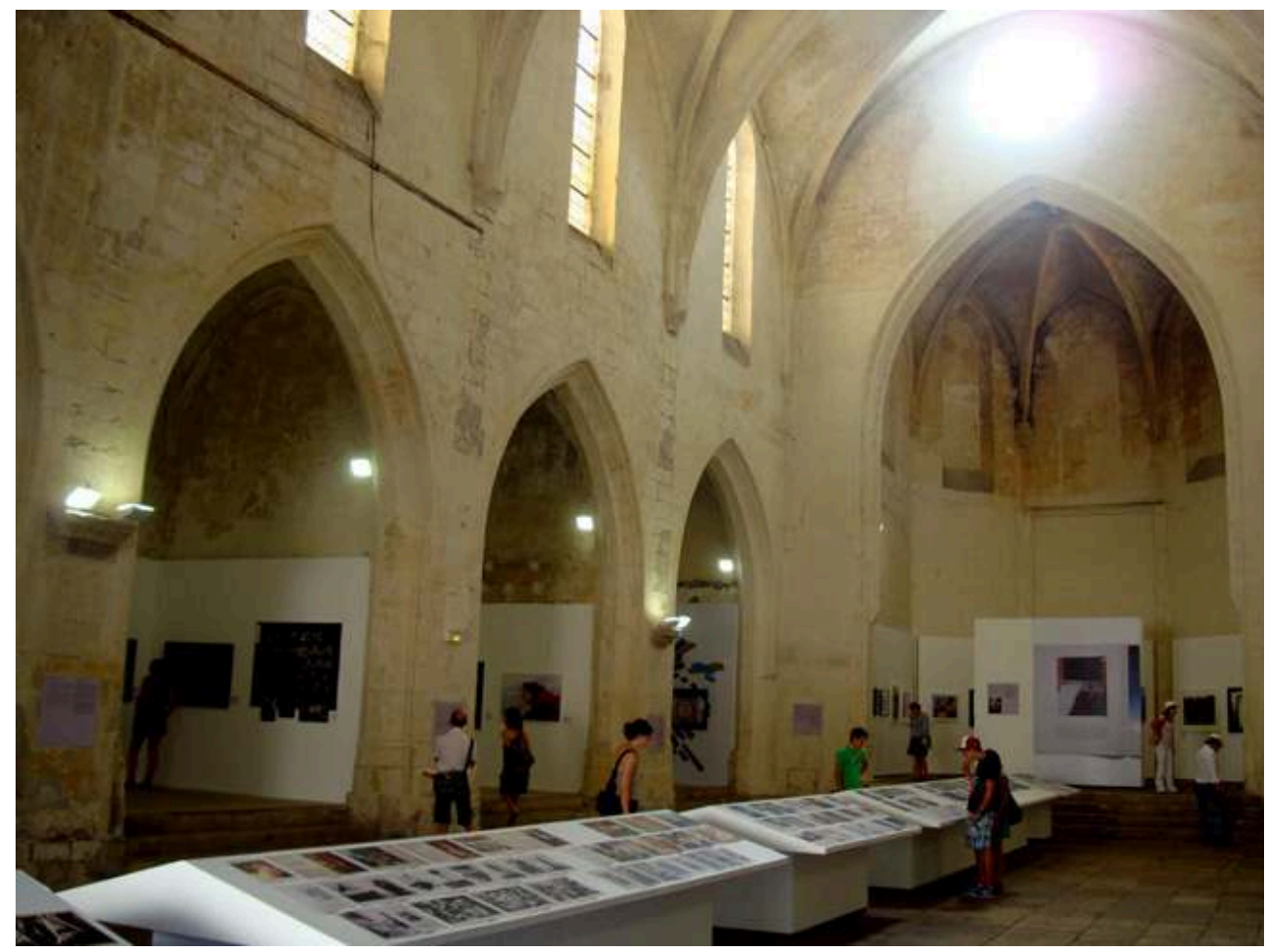

"Les photographies du New York Times Magazine », Chapelle Sainte-Anne, Arles,

4 juillet - 4 septembre 2011

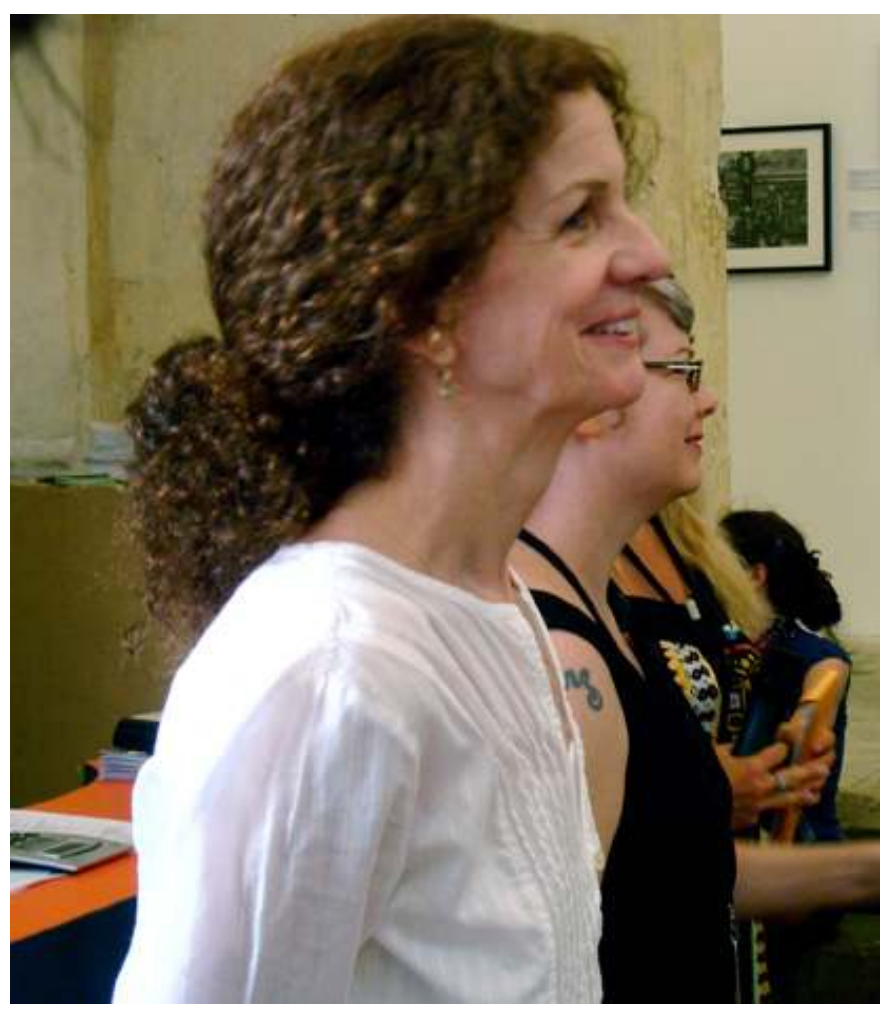

Kathy Ryan - directrice de la photographie du New York Times Magazine -

et Lesley A. Martin, commissaires d'exposition. Visite d'exposition, le 7 juillet 2011.

Dans le cadre d'une thématique placée sous le signe du "document $»^{1}$, les Rencontres d'Arles de l'été 2011 offrent une exposition inédite en l'honneur des trente ans du New 
York Times Magazine. Onze installations dans la chapelle Sainte-Anne d'Arles permettent au visiteur de mesurer la diversité photographique à l'œuvre dans le supplément hebdomadaire du célèbre quotidien nord-américain ${ }^{2}$. Photojournalisme, photographie de mode, de sport, portraits d'acteurs, commandes spécifiques et portfolios sont présentés avec un souci éditorial qu'il n'est plus rare de trouver dans la scénographie des expositions de photographies. Ainsi, en complément des tirages de grand ou moyen format, il est permis de voir certaines planches contacts, de lire des correspondances ou des témoignages de photographes, de directeurs artistiques, de rédacteurs, des extraits de carnets de voyage. Puis, tout au long de la nef de l'église, un fac-similé des pages du magazine présente l'intégralité du reportage publié. Le texte d'introduction de chaque installation éclaire sur les conditions de production des images, leur contexte historique, la demande faite au photographe, son intention ou celle du rédacteur en chef $^{3}$.

2 Le regard se perd d'abord un peu dans les dédales des expositions dont la plupart sont accrochées dans les chapelles latérales, celle sur Times Square figurant dans le chœur de l'église. Organisées par thèmes - «Mode et mélange de style ", "Une réponse au 11 septembre », "Mission: Times Square », «Grands acteurs " - ou bien centrées sur l'œuvre de plusieurs photographes aux missions et aux styles très différents - Gilles Peress, Ryan McGinley, Lynsey Addario, Simon Norfolk, Paolo Pellegrin, Gregory Crewdson, Sebastião Salgado -, les installations se laissent d'abord voir en totalité puis l'on ne peut s'empêcher de revenir sur les plus troublantes ou les plus marquantes qui sont autant de coups de cœur photographiques.

3 Kathy Ryan ${ }^{4}$, commissaire de l'exposition - en partenariat avec Lesley Martin d'Aperture Foundation - et rédactrice en chef de la photographie au New York Times Magazine, a eu l'occasion de donner durant la semaine d'ouverture des Rencontres (4-10 juillet) quelques clés de lecture pour mieux cerner l'objectif que se donne le magazine à la recherche de la «bonne photo» ou du «bon reportage " . Outre des qualités essentielles, relatives à la capacité à saisir et à jouer avec la lumière, la couleur ou bien le noir et blanc, à un sens de la composition aigu et à une maitrise technique exemplaire pour exprimer visuellement une idée, un mot revient sans cesse, celui de l'originalité du regard du photographe, quel que soit par ailleurs son sujet: «People have a certain kind of individual point of view and the best photographers have that point of view very focused and a very strong idea of what they want to find, either in a character portrait or out in the field, covering a story $"^{6}$.

4 La quête du photographe devient celle de la rédactrice à la recherche d'un angle de vue inédit sur un sujet : " Every assignment is different but for many assignments it's about imagination. Who's got a point of view that would just go against expectation on the subject? Because whenever we can delight readers when they open the magazine and find something they did not expect, that's number one ${ }^{7}$. C'est cette question qui préside à une technique que Kathy Ryan affectionne particulièrement, celle du « crossassigning ». Le fait de confier à des photographes connus pour leur pratique artistique singulière une mission dans un univers qui leur est peu habituel apparait comme une source de créativité souvent encouragée dans le magazine. "Many artists leap at the chance because it's just something different and creative. People love to be asked to do something different $»^{8}$. Il en est ainsi d'une commande dans l'univers de la mode confiée notamment à Lee Friedlander et Nan Goldin'. 
5 À sa manière de street photographer, Lee Friedlander organise le chaos des coulisses de la mode en créant des motifs nouveaux, pareils au grouillement frénétique d'une ruche où s'affairent les ouvrières - maquilleuses, coiffeuses, habilleuses, photographes...- sans jamais perdre de vue les missions de chacun des protagonistes. Le motif des mains est omniprésent et semble dire la prédation à l'œuvre lorsque l'appareil cible de très jeunes mannequins dont certains paraissent des enfants-impression accentuée lorsque les prises de vue sont réalisées en plongée.

6 Faisant face aux clichés en noir et blanc de Lee Friedlander, surgissent les couleurs tour à tour chaudes ou froides du reportage de Nan Goldin sur un jeune mannequin à la dérive, James King ${ }^{10}$. Lolita fragile à la beauté troublante, James inspire la photographe, qui admet reconnaître en elle les comportements autodestructeurs de sa jeunesse : "James and I connected very quickly. I understood her, in some ways, because of my own youth and history. She was very self-destructive. I had this kind of caring for her, as if she were my child, and I really wanted to help her $»^{11}$. Elle inspire aussi la romancière Jennifer Egan, qui travaille en binôme avec la photographe dans ce pictureessay, et dont le texte de couverture est accrocheur, en phase avec les clichés : « She has a look that's earned her runway jobs, magazine covers, tens of thousands of dollars and a shot at celebrity. All she's lost is her youth.» ${ }^{12}$ En août 2011, seize ans plus tard, James, devenue actrice, s'exprime sur cette période de sa vie dans les colonnes du magazine qui, fait rare, donne la parole aux sujets photographiés : «I was working with these masters in fashion and photography and learning from brilliant, creative people from around the world. (...) I felt like some people wanted a piece of me, wanted to take something from me. I felt they wanted to sexualize me $»^{13}$. La photo de couverture ${ }^{14}$, aux tons chauds et lumineux, toute en tension, offre un flagrant contraste avec nombre d'images de la série prises dans un contexte le plus souvent informel: le modèle, allongé sur le ventre, les jambes repliées dans les coulisses d'un défilé pour Karl Lagerfeld, fume une cigarette ; James est maquillée, parfaitement apprêtée, elle se sait regardée. À l'opposé de cette image, assise sur les genoux de son petit ami Kyle, elle laisse voir un corps de pantin désarticulé, las, ses paupières sont closes, son expression désabusée, elle est épuisée, le champagne coule à flots, et le spectateur reconstruit malgré lui une histoire dont il ignore tout, mais que ces clichés, dans leur force évocatrice, poussent irrésistiblement à inventer.

7 Dans un registre radicalement différent, l'espace d'exposition consacré au 11 septembre 2001 à New York permet d'envisager le processus photographique de façon totale, de la survenue d'un événement traumatisant à la publication d'un reportage de 106 pages - soit l'intégralité du magazine -, douze jours ${ }^{15}$ après la diffusion par la presse du monde entier de quantités de clichés dont certains marquent encore la mémoire collective. Comment, face à un événement à l'onde de choc émotionnelle si forte, aux répercussions politiques planétaires, répondre à l'impératif maintes fois répété de K. Ryan : «to break the expected pattern of representation, with something deeper ", "to find an unexpected approach », «trying every week something fresh, new, original $»^{16}$ ?

8 La mise en scène de cette partie d'exposition est remarquable dans son souci de communiquer au visiteur les éléments d'appréciation d'un processus à l'œuvre, dans un climat de déréliction, couplé à une conscience professionnelle exacerbée par des circonstances douloureuses. Pendant plusieurs jours, l'équipe du magazine travaille sans relâche. C'est en ces termes qu'Arthur O. Sulzberger, Jr., directeur du groupe New 
York Times Company, s'adressera par courrier aux équipes qui réussissent à sortir le numéro du journal au lendemain de la tragédie, dans des conditions matérielles et psychologiques extrêmement difficiles: "To our remarkable reporters, editors, photographers, graphic artists, and newsroom support staff, you have the gratitude and admiration of all of us. $»^{17}$ Une des questions centrales qui occupe la rédaction est l'image de couverture ; elle sera l'aboutissement d'un travail complexe de réflexion et de trucage photographique visant à ériger deux faisceaux lumineux en lieu et place des tours jumelles effondrées. La photographie publiée, Phantom Towers, a été conçue par les artistes Paul Myoda et Julian LaVerdiere à partir d'une photo originale de Fred Conrad. Elle est retouchée sur Photoshop, afin que le faisceau de lumière blanche, qui traversait horizontalement l'image prise en début de soirée d'une barge sur le fleuve Hudson, puisse être orienté à la verticale puis dupliqué, sans avoir recours à un montage composite. «It's an emotional response more than anything. Those towers are like ghost limbs; we can feel them even though they are not there anymore. [...] We realized that the best thing we can do to help is an artistic gesture that might offer consolation or a sense of security or hope $\aleph^{18}$, confie Julian LaVerdiere dans les colonnes du magazine. Un an plus tard et de manière désormais rituelle, l'idée prendra corps, et de véritables colonnes de lumière (88 projecteurs), Tribute of Light, s'élèveront à côté de l'emplacement du World Trade Center, en guise de mémorial pour commémorer le 11 septembre ${ }^{19}$.

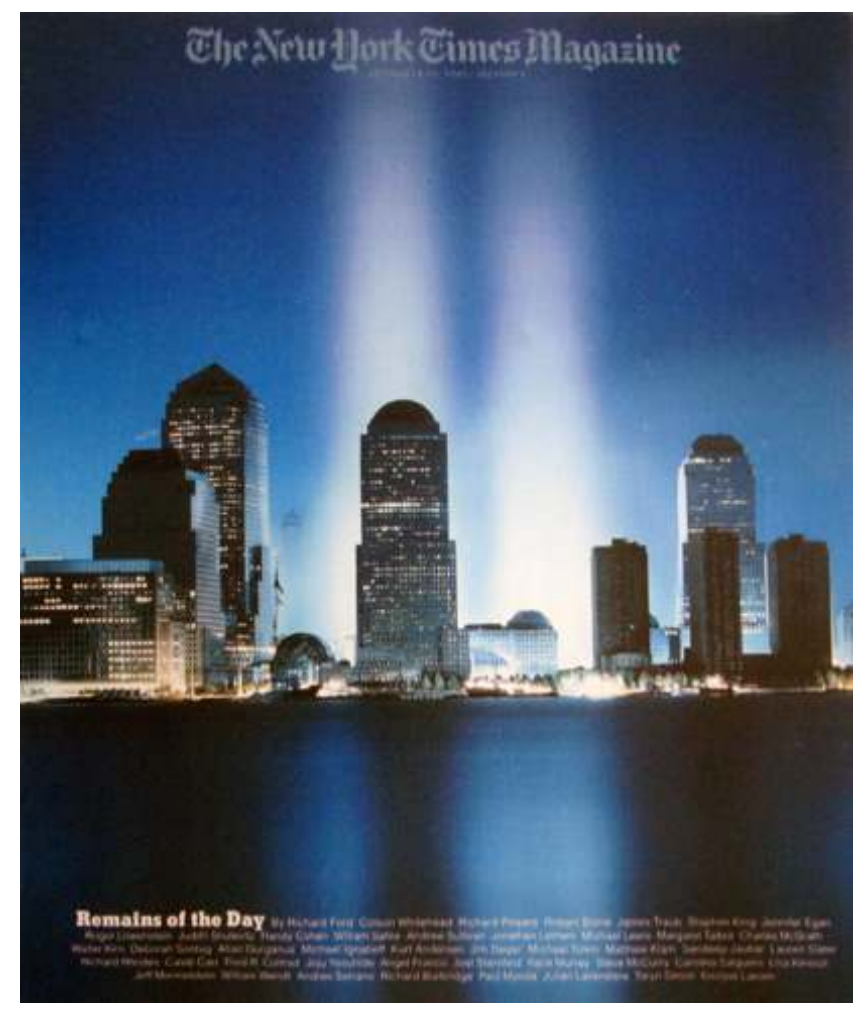

Couverture du New York Times Magazine, 23 septembre 2001.

Outre les photographies citées, la vision de l'Iran de Gilles Peress au tout début des années 80, les puits de pétrole en feu du Koweït de Sebastião Salgado, le Darfour de Paolo Pellegrin ou dans un tout autre registre ses photos d'acteurs qui défient les codes de représentation des clichés de célébrités - Leonardo DiCaprio ou Kate Winslet -, l'univers hoppérien de Gregory Crewdson avec sa «Dream House» et bien d'autres encore, montrent à quel point les grands noms de la photographie ont contribué à la 
notoriété du New York Times Magazine - et inversement. Peut-être aurait-on apprécié une information ou une place particulière consacrée à l'émergence de nouveaux talents, passés - photographes devenus célèbres, mais découverts par le magazine - ou contemporains, d'autant que le magazine dit en faire un axe majeur de sa politique, par la voix de sa directrice de la photographie : « To be successful, a magazine has to be a mix of big names and totally unknown. A big part of the photo editor is discovery, a lot of work with students of the ICP ${ }^{20}$. We are looking for new work all the time, for new looks bringing fresh points of view» ${ }^{21}$. L'exposition n'en reste pas moins très riche, en termes de contenu mais aussi dans ses modalités de présentation.

Liens : http://www.nytimes.com/pages/magazine/index.html

http://www.rencontres-arles.com

11 Publication : The New York Times Magazine Photographs, Edited by Kathy Ryan. Preface by Gerald Marzorati, Aperture, 2011.

Crédits photographiques : figs. 1 et 2 Anne Lesme ; fig.3, avec l'aimable autorisation du New York Times Magazine.

\section{NOTES}

1. Avec « Robert Capa, Chim (David Saymour), Gerda Taro : La valise mexicaine ».

2. Exposition complétée par une présentation de commandes photographiques et articles de fond importants qui ont jalonné les 30 ans du magazine, au cloître Saint-Trophime. Fac-similés présentés sur deux longues rangées.

3. Voir l'interview de Kathy Ryan accordée à La Lettre de la Photographie.com: http:// lalettredelaphotographie.com/archives/by_date/2011-07-06/3243/kathy-ryan-and-the-newyork-times-magazine

4. Sous sa direction, le magazine a remporté de nombreux prix : Pictures of the Year, World Press Photo, The Society of Publication Designers ou The Overseas Press Club. Kathy Ryan est rédactrice en chef de la photographie au New York Times Magazine depuis 1985, après avoir travaillé à l'agence Sygma. Elle a remporté le prix Canon Best Picture Editor of the Year au festival Visa pour l'Image de Perpignan en 1997 et a été nommée Picture Editor of the Year aux Lucie Awards en 2003.

5. Visite d'exposition avec les commissaires Kathy Ryan et Lesley A. Martin, le 7 juillet 2011 ; Débat-rencontre: «La photographie peut-elle faire vendre des journaux en 2011 ? (New York Times Magazine, Télérama, le Monde Mag), avec Kathy Ryan, le 8 juillet 2011.

6. Kathy Ryan, http://www.charlierose.com/view/interview/6151(page consultée en août 2011).

7. Kathy Ryan, 7 juillet 2011, Arles.

8. Kathy Ryan, 7 juillet 2011, Arles.

9. Ainsi qu'à Jeff Koons, Roger Ballen, Alfred Seiland et Malick Sidibé.

10. La série s'est vendue à $\$ 37,087$ le 28 juin 2002 (estimation: entre $\$ 22,545-\$ 30,060$ ) chez Christies, http://www.christies.com/LotFinder/lot_details.aspx?intObjectID=1827218 (page consultée en août 2011).

11. Propos cités dans l'exposition.

12. « At 16, a Model's life », New York Times Magazine, 4 février 1996. 
13. 18 août 2011, http://www.nytimes.com/interactive/2011/08/21/magazine/ Mag-21Thinking.html?ref=magazine\#6 (lien actif en août 2011)

14. http://www.mocp.org/collections/permanent/goldin_nan.php (lien actif en août 2011).

15. Le 23 septembre 2001.

16. Kathy Ryan, 7 et 8 juillet 2011, Arles.

17. Reproduit dans l'exposition.

18. "Filling the Void", New York Times Magazine, 23 September 2001.

19. La première installation a lieu entre le 11 mars et le 14 avril 2002 et la photographie fait la Une du New York Times le 12 mars.

20. ICP : International Center of Photograpy, New York City. ICP School : http://www.icp.org/ school (lien actif en août 2011).

21. Kathy Ryan, 8 juillet 2011, Arles.

INDEX

Thèmes : Trans'Arts 